\title{
UNIDAD DE ANALISIS PARA EL ESTUDIO DE LAS ORGANIZACIONES
}

Hernán Alvarez Londoño*

El artículo presente tiene por objeto presentar un marco conceptual útil para el estudio de las organizaciones y de su d:námica social interna y externa.

Comprender a una organización implica no sólo tener un conocimiento claro de si: pasado sino, también, de su situación actual, así como de sus amenazas $y / o$ posibilidades futuras.

Es por esto que el marco conceptual que aquí se presenta está conformado: en primer lugar, por el proceso histórico de la organización, el cual es el punto de partida para la comprensión de la misma; y en segundo lugar por aquellos elementos con los cuales es posible realizar el estudio de la situación actual y de sus amenazas y/o posibilidades futuras. Estos elementos son: el entorno social, los procesos organizacionales y los seres humanos (figura No. 1).

\section{El proceso histórico}

Analizar el proceso histórico de una organización consiste en conocer y comprender la "esencia" de la misma. Es decir, conocer y comprender las causas que la originaron y, fundamentalmente los por qués de su desarrollo, en una forma y no en otra, durante las diferentes fases por las cuales ha pasado desde su génesis hasta nuestros días.

Ingeniero Civil, Magister en Administración y en Investigación de Operaciones del Instituto Tecnológico de Monterrey, seminarios de Desarrollo Organizacional y Efectividad Gerencial del IDO - Caracas. Profesor del Departamento de Administración de la Universidad del Valle en el ára de Administración y Desarrollo Humano. 

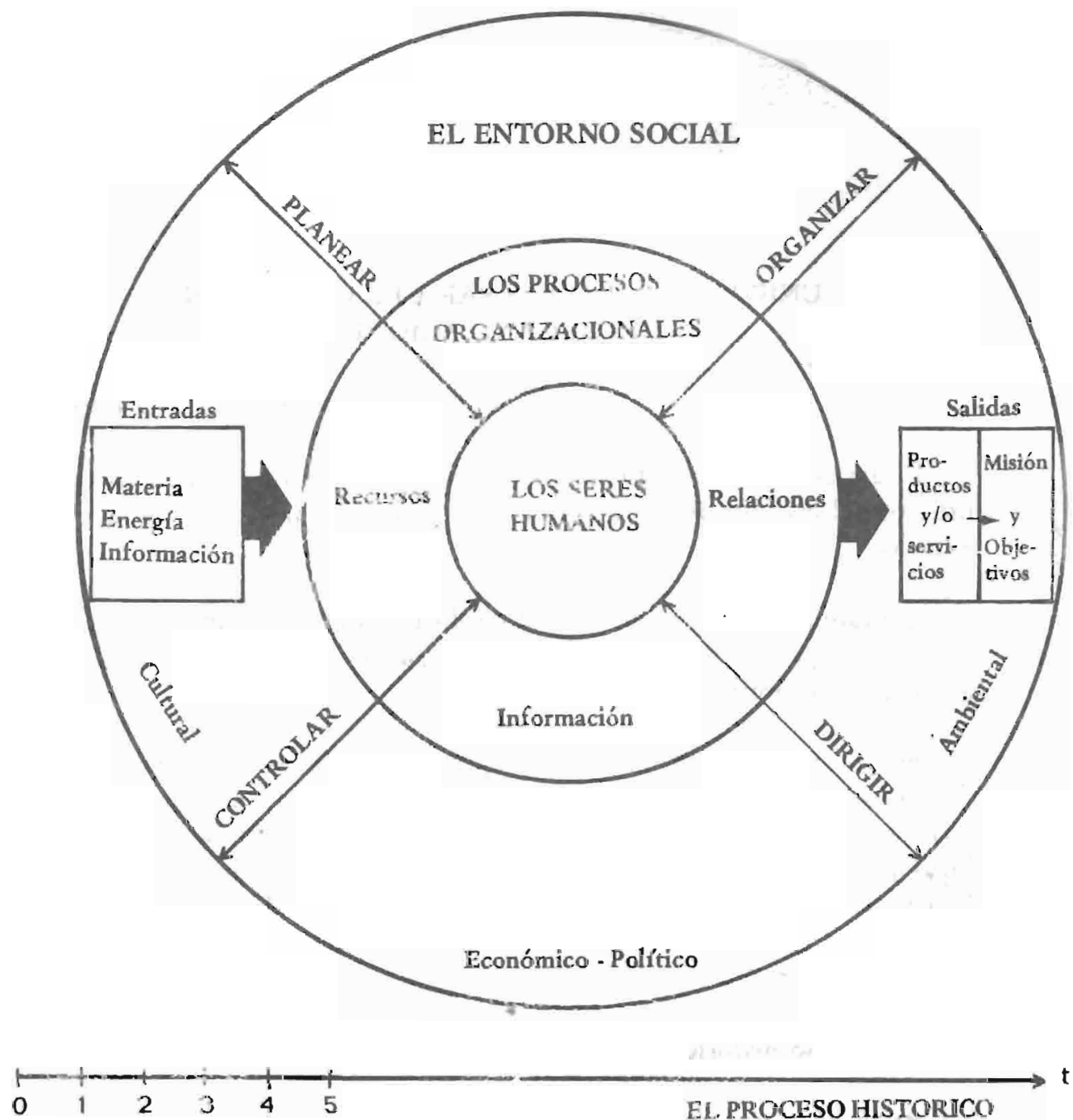

Figura No. 1

UNIDAD DE ANALISIS PARA EL ESTUDIO DE LAS ORGANIZACIONES 


\section{La situación actual}

El estudio de la situación actual de la organización puede realizarse a través de análisis del entorno social, los procesos organizacionales y los seres humanos.

\section{a. El entorno social}

El entorno social es el suprasistema global con el cual la organización se encuentra en inter-relación permanerte, en forma directa o indirecta, el cual está integrado por los sistemas cultural, económico-político y ambiental.

1) El sistema cultural está conform $x$ do por aquellos aspectos que determinan la forma de vida de una sociedad, tales como valores, roles y normas de comportamiento del individuo, de la familia, de las organizaciones y de la sociedad, así como por las expresiones artísticas científicas y tecnológicas, las cuales son el resultado de la acción del hombre sobre la naturaleza.

2) Al sistema económico-político lo definen los orupos humanos, organizaciones e instituciones que participan en el campo de los recursos, de los mercados y de la política, en relación directa y recípruca con la organización, o indirectamente a través del Estado en el establecimiento de las normas que hacen referencia a las relaciones en la sociedad y desde luego al funcionamiento de las organizaciones; $y$

3) Al sistema ambiental lo integran los recursos naturales renovables y no renovables, a saber: aire, agua, bosques, animales, yacimientos, minas, etc. Aunque este sistema forma realmente parte del sistema económico, se le ha querido destacar dada la importancia que tiene para la sociedad y su futuro.

Una conformación más detallada del entorno social de las organizaciones podría contener los siguientes sistemas: cultural, demográfico, económico, político, jurídico, ecológico y organizacional.

Sin embargo, sin importar cuál sea la subdivisión que se haga de dicho entorno, el análisis de éste requiere no solament conocer y comprender cada uno de tales subsistemas, sino también los cé bios que tienen lugar en el entorno mismo, en su estructura, en sus relaciones, en sus procesos, pues como bien lo señalan Emery y Trist: "La estructura del medio ambiente (entorno social) cambia independientemente de la organización 
y por lo tanto las relariones entre los diferentes elementos del medio (The causal texture of environments), deben ser estudiadas en ellas mismas"1. Naturalmente, el estudio debe complementarse con el análisis del impacto que tienc el entorno en la organización y viceversa.

\section{b. Los procesos organizacionales}

Con miras a ąlcanzar su misión y objetivos en las organizaciones se realizan procesos de diferente naturaleza, los cuales pueden estar dirigidos hacia aspectos internos y/o externos de la organización. Es decir, procesos de producción, de mercadeo, de investigación, contable-financieros, de selección y capacitación de personal, etc.

Cada uno de estos procesos puede analizarse teniendo presente las diferentes partes que lo conforman, la dinámica del proceso en sí mismo, su contribución a los objetivos organizacionales, la forma como están administrados en cuanto a su gestión y dirección, etc.

En términos generales, los procesos organizacionales están conformados por los siguientes aspectos:

1) Los recursos, los cuales comprenden todos aquellos elementos distintos al hombre: naturales, económicos, tecnológicos, materiales, etc.

2) La información, la cual es indispensable tanto para el buen desarrollo del proceso como para la toma de decisiones; y

3) Las relaciones, entendidas fundamentalmente entre seres humanos, formales e informales, por lo tanto, pero siempre necesarias para el trabajo.

La parte fundamental del análisi; de todos los procesos organizacionales la constituye, sin embargo, la forma como todos ellos se integran en un todo con el fin de transformar las entrádas a la organización (materia, energía e información), en salidas (productos y/o servicios) que le permitan alcanzar, efectivamente, su misión y sus objetivos.

c. Los seres humanos

Los seres humanos deben entenderse como la célula vital de las organi-

(1) F. E. Emery and E. L. Trist. "The causal Texture of Organizational Environments", Human Relations. No. 18,1965 , pp. 21-31. 
zaciones. Son ellos, en conjunto, quienes en definitiva alcanzan los fines y y objetivos organizacionales y para quienes su trabajo representa una parte importante en la satisfacción de sus necesidades.

Esta parte del estudio debe centrarse, fundamentalmente, en el análisis del comportamiento humano en la organización, teniendo presente que éste depende de diferentes causas, a saber:

1) De cada uno de los individuos, pues como seres en desarrollo que son, tienen diferentes valores, actitudes, conocimientos, experiencias, necesidades, aptitudes, etc.

2) De cada uno de los grupos organizacionales y de su dinámica interna, entendiendo como tal: el estilo de dirección, los sistemas de toma de decisiones y solución de conflictos, las normas, los roles, etc.

3) Del conocimiento que se tenga de la organización, de su misión y sus objetivos, así como del tipo de estructura, del clima organizacional, de las políticas organizacionales, del medio ambiente externo, etc., y

4) De la capacitación que tengan los individuos para poder realizar sus funciones, primordialmente las administrativas (planear, organizar, dirigir y controlar), con miras a una acción eficaz, finalmente, en la organización.

El estudio de la organización debe concluir con el análisis conjunto de todos los elementos anteriormente mencionados. Es decir, integrando a lo administrativo, el análisis de sus relaciones, sus interdeperdencias, sus contradicciones, etc., de la forma más clara y objetiva posible, hasta obtener una visión real de la situación actual de la organización, así como de sus posibilidades y amenazas futuras.

\section{BIBLIOGRAFIA}

Hall H., Richard. Organizations: Structure and Process. 2th edition, Englewood Cliffs, N. Y., U. S. A., Prentice Hall, 1977.

Hodge, Billy J. y Hebert J. Hohnson. Administración y organización. Buenos Aires, Editorial El Ateneo, 1975.

Gutiérrez P., Gerardo. Administración: ciencia y arte. Bogotá, Universidad Externado de Colombia, 1980. 
Robbins R., Stephen. Organizational Behavior: Concepts and Controversies. Englewood Cliffs, N. Y., U. S. A., Prentee Hall, 1979.

Sevilla, Andrés. Análisis de las organizaciones. Documento mimeografiado, Cali, Universidad del Valle, Departamento de Administración. 